() А.С. Северина', И.И. Ларина' ${ }^{1 *}$, А.С. Шутова', М.Ш. Шамхалова', И.В. Дмитриев², А.В. Пинчук ${ }^{2,3}$, С.В. Арзуманов ${ }^{4}$, М.В. Шестакова'

'Национальный медицинский исследовательский центр эндокринологии, Москва

${ }^{2}$ Научно-исследовательский институт скорой помощи им. Н.В. Склифосовского, Москва

${ }^{3}$ Научно-исследовательский институт организации здравоохранения и медицинского менеджмента, Москва

${ }^{4}$ Научно-исследовательский институт урологии и интервенционной радиологии им. Н.А. Лопаткина - филиал

ФГБУ «НМИЦ радиологии», Москва

Сочетанная трансплантация почки и поджелудочной железы (СТПиПЖ) в современном мире выступает наиболее перспективным методом лечения пациентов с сахарным диабетом 1 типа (СД1) и терминальной стадией диабетической нефропатии (ДН). Результатом успешной трансплантации является одномоментная ликвидация уремической интоксикации и гипергликемии - ведущего триггера сосудистых осложнений. Эугликемия в этом отношении, являясь важным метаболическим изменением у пациентов после операции, остается лишь одним из факторов удовлетворительного функционирования почечного аллотрансплантата. В случае возобновления заместительной почечной терапии (ЗПТ) посредством диализа у реципиентов после СТПиПЖ вопросы ведения и мониторинга состояния трансплантата поджелудочной железы (ТПЖ) остаются открытыми. Отдельное внимание к функции ТПЖ обусловлено не только потенциальным риском развития хирургических осложнений, но и некоторой вероятностью рецидива сахарного диабета 1 типа с необходимостью возобновления инсулинотерапии. У пациентов с сохранной функцией обоих трансплантатов превалирующее значение приобретает оценка динамики диабетических осложнений в целом. Результаты немногочисленных исследований в этом отношении остаются противоречивыми. Таким образом, клинические варианты могут быть непредсказуемо разнообразными и требовать не только поиска первопричины, но и оптимизации реабилитационной тактики, даже в случае достижения ожидаемой компенсации.

КЛЮЧЕВЫЕ СЛОВА: сочетанная трансплантачия почки и поджелудочной железы; сахарный диабет 1 mипа; трансплантат; эугликемия; клинический случай

\title{
SIMULTANEOUS PANCREAS-KIDNEY TRANSPLANTATION IN TYPE 1 DIABETES MELLITUS. CLINICAL OPTIONS
}

(c) Anastasia S. Severina' ${ }^{1}$ Irina I. Larina ${ }^{1 *}$, Aleksandra S. Shutova1', Minara Sh. Shamkhalova', Ilya V. Dmitriev², Alexey V. Pinchuk ${ }^{2,3}$, Sergey V. Arzumanov ${ }^{4}$, Marina V. Shestakova ${ }^{1}$

\section{${ }^{1}$ Endocrinology Research Centre, Moscow, Russia}

${ }^{2}$ Sklifosovsky Research Institute for Emergency Medicine, Moscow, Russia

${ }^{3}$ Research Institute for Healthcare Organization and Medical Management, Moscow, Russia

${ }^{4}$ N.A. Lopatkin Research Institute of Urology and Interventional Radiology - branch of the National Medical Radiological

Research Centre of the Ministry of Healthcare, Moscow, Russia

Simultaneous pancreas-kidney transplantation (SPKT) is the most promising treatment option for patients with type 1 diabetes mellitus (T1DM) and end-stage renal disease (ESRD) due to diabetic nephropathy (DN). Successful SPKT eliminates uremic intoxication and hyperglycemia - the leading trigger of vascular diabetic complications. Therefore, euglycemia is an important metabolic change in patients after surgery and remains only one of the factors for the saved renal allograft functioning. In the case of resuming renal replacement therapy by dialysis after SPKT, the management and monitoring of the pancreatic graft remains open. Special attention to the pancreatic graft's function is due to both the potential risk of surgical complications, and some probability of T1DM relapse with the need to resume insulin therapy. In patients with saved function of both transplants, the assessment of the dynamics of diabetic complications in general becomes more important. The results of few studies in this regard remain contradictory. Thus, clinical options can be unpredictably diverse and require not only search for the root cause, but also optimization of rehabilitation tactics, even if the expected results are achieved.

KEYWORDS: simultaneous pancreas-kidney transplants; diabetes mellitus type 1; graft; euglycemia; case report 
Сочетанная трансплантация почки и поджелудочной железы (СТПиПЖ) является одним из видов мультиорганной трансплантации, которую проводят пациентам с сахарным диабетом 1 типа (СД1) и терминальной стадией диабетической нефропатии (ДН). После СТПиПЖ уменьшение смертности по сравнению с категорией пациентов, получающих диализ, наблюдается главным образом вследствие трансплантации почки. Основным преимуществом операции является улучшение качества жизни за счет отсутствия необходимости диализа, частого мониторинга уровня глюкозы, инсулинотерапии, риска гипогликемий, а также вероятности если не регресса, то по меньшей мере стабилизации сосудистых осложнений.

В целом достаточно низкая трансплантационная активность в случае СТПиПЖ обусловлена дефицитом донорских органов, а также, главным образом, небольшим числом пациентов с сохранной функцией обоих трансплантатов чаще в позднем послеоперационном периоде, что отчасти является следствием несовершенной на сегодняшний день системой отдаленного ведения этих пациентов.

Трансплантация поджелудочной железы (ПЖ) как изолированно, так и в комплексе с почкой представляет собой сложное хирургическое вмешательство с высоким риском как ранних, так и поздних послеоперационных осложнений. Вследствие совершенствования хирургической техники, иммуносупрессивной терапии, подбора донорского материала выживаемость пациентов и трансплантатов ПЖ существенно увеличилась в последние годы и в настоящее время составляет 95\% и 85\% соответственно [1]. Средний период полужизни трансплантата ПЖ (ТПЖ) в настоящее время составляет 16,7 года, достигая наиболее длительной продолжительности среди экстраренальных трансплантатов [2] и практически соответствует таковой почечных трансплантатов от посмертных доноров [3].

Лидирующей причиной смерти пациентов в раннем посттрансплантационном периоде являются инфекционные осложнения, тогда как в отдаленном периоде преобладают сердечно-сосудистые события. Другими значимыми причинами смерти являются кровотечения и новообразования [1].

Успешная СТПиПЖ позволяет существенно улучшить качество жизни пациентов с СД1 и снизить экстремально высокий риск сердечно-сосудистых событий при проведении заместительной почечной терапии (ЗПТ) посредством процедур диализа. Потеря трансплантата оказывает существенное воздействие на относительный риск (ОР) смерти реципиента. При потере пересаженной почки ОР смерти реципиента увеличивается практически в 11 раз. Когда происходит потеря ТПЖ, ОР смерти реципиента возрастает приблизительно в 3 раза [1].

В данной статье представлено описание клинических случаев различного развития событий после успешной СТПиПЖ: утрата функции ТПЖ на фоне сохранения удовлетворительной почечной функции, потеря почечного трансплантата с возвратом к ЗПТ диализом при сохранной функции ТПЖ и длительная (более 11 лет) удовлетворительная функция обоих трансплантатов.

\section{ОПИСАНИЕ КЛИНИЧЕСКИХ СЛУЧАЕВ}

Пациент Н., 63 года, СД1 был диагностирован в 1981 г. (с 25-летнего возраста, в течение 37 лет).

На рис. 1 схематично представлено течение заболевания.

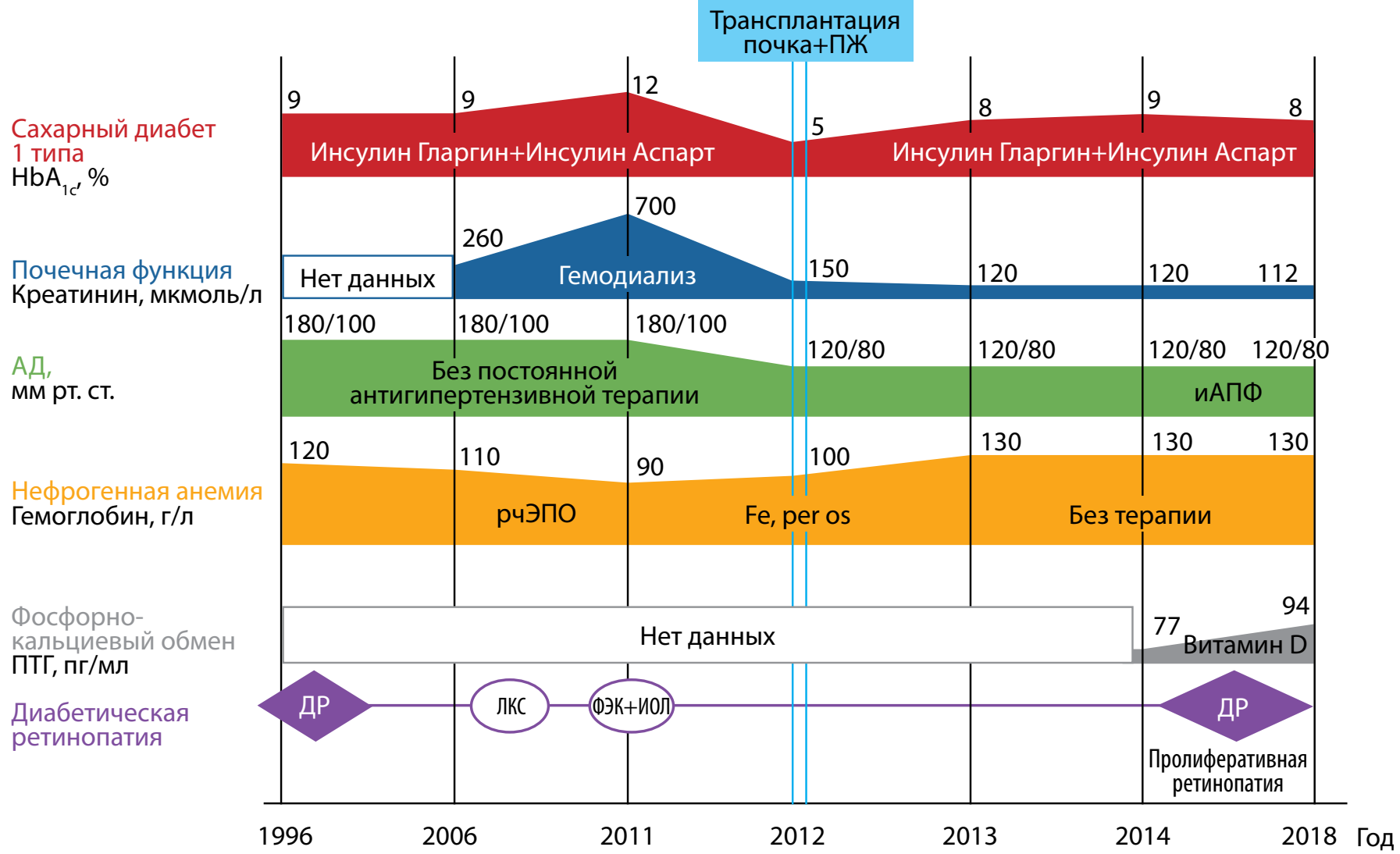

Рис. 1. Графическое представление течения заболевания пациента $\mathrm{H}$.

$\mathrm{HbA}_{1 c}$ - гликированный гемоглобин; ПтГ - паратгормон; рчЭПО - препараты рекомбинантного человеческого эритропоэтина; Fе рег оs пероральные препараты железа; ДР - диабетическая ретинопатия; ЛКС - лазеркоагуляция сетчатки; ФЭК+ИОЛ - факоэмульсификация катаракты+имплантация интраокулярной линзы; иАПФ - ингибиторы ангиотензинпревращающего фермента. 
На всем протяжении заболевания стойкой компенсации углеводного обмена не достигалось, пациент не был привержен к лечению, в школе самоконтроля для больных СД1 не обучался, рекомендации врачей не соблюдал. Данный факт привел к развитию микрососудистых осложнений приблизительно через 15 лет от момента дебюта СД1. Обращают на себя внимание поздняя диагностика ДН, уже на стадии почечной недостаточности, а также отсутствие адекватной нефропротективной терапии (регулярного приема ингибиторов ангиотензинпревращающего фермента (иАПФ)), что привело кдостаточно быстрому развитию терминальной стадии хронической болезни почек (ХБП). 05.01.2012 г. была проведена додиализная одномоментная аллотрансплантация трупных почек в левую подвздошную область и ПЖ справа с использованием методики мочепузырного дренирования (отведение экзокринного секрета в мочевой пузырь). Первичная функция обоих трансплантатов была удовлетворительной. Эугликемия была отмечена на 3-и сутки, нормализация уровня азотистых шлаков - к 5-м суткам после операции. Была инициирована стандартная трехкомпонентная иммуносупрессивная терапия, которую пациент получает по настоящее время. Впоследствии, в течение 1 года, со слов пациента, в связи с диетическими погрешностями, а также несоблюдением рекомендаций по регулярному контролю концентрации иммуносупрессивных препаратов в крови появилась и постепенно нарастала потребность в инсулине. В конечном итоге через 1 год после пересадки это привело к необходимости возобновления постоянной интенсифицированной инсулинотерапии; колебания гликированного гемоглобина составили 8,4-9,2\%.
Состояние почечного трансплантата удовлетворительное по настоящее время (расчетная скорость клубочковой фильтрации (рСКФ) ЕРІ 60 мл/мин/1,73 м², соотношение альбумин/креатинин в разовой порции мочи 0 мг/ммоль).

Таким образом, особенностью данного клинического случая является стремительное развитие дисфункции ТПЖ на фоне удовлетворительной функции почечного трансплантата. К сожалению, более углубленного обследования пациента с оценкой уровня антител, биопсией не проводилось. В результате СТПиПЖ у пациента поддерживается удовлетворительная функция пересаженной почки на протяжении 8 лет после трансплантации, на фоне чего отмечаются улучшение течения артериальной гипертензии, нормализация уровня гемоглобина. Выявленный посттрансплантационный гиперпаратиреоз может быть обусловлен дефицитом витамина D, снижением реабсорбции кальция в кишечнике и развитием дефицита половых гормонов на фоне постоянного приема иммуносупрессивной терапии. Отсутствие значительного числа анамнестических данных не позволило нам оценить степень персистенции минеральных и костных нарушений при ХБП у данного пациента.

Пациентка Б., 37 лет, диагноз СД1 был установлен в 1993 г. (в возрасте 11 лет, длительность заболевания 26 лет).

Схематично течение заболевания представлено на рис. 2.

На всем протяжении заболевания отмечалась крайне низкая приверженность пациентки к лечению, несоблюдение рекомендаций врачей, в связи с чем на всем протяжении заболевания компенсации

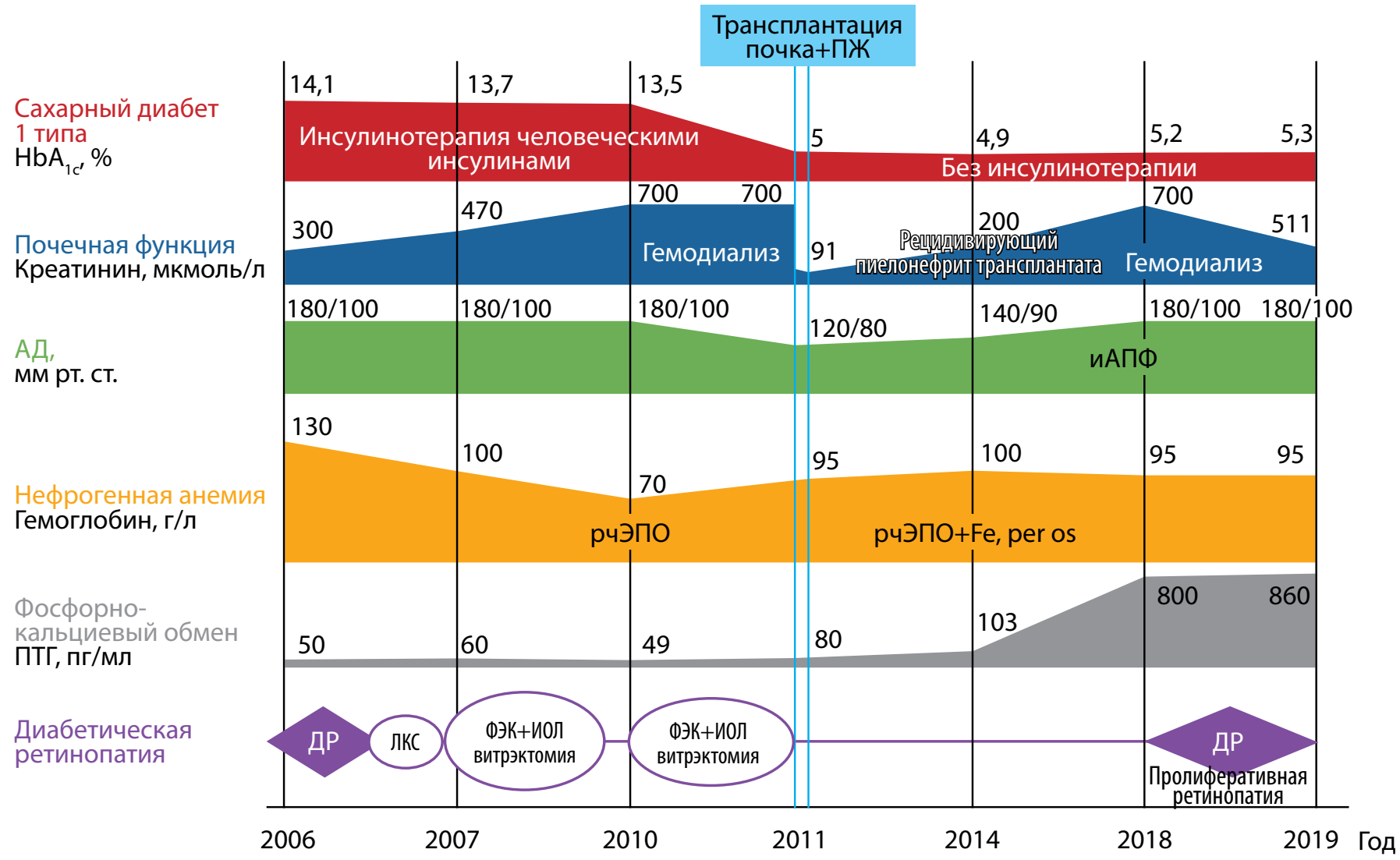

Рис. 2. Графическое представление течения заболевания пациентки Б.

$\mathrm{HbA}_{1 c}$ - гликированный гемоглобин; ПТГ - паратгормон; рчЭПО - препараты рекомбинантного человеческого эритропоэтина; Fe per os - пероральные препараты железа; ДР - диабетическая ретинопатия; ЛКС - лазеркоагуляция сетчатки; иАПФ - ингибиторы ангиотензинпревращающего фермента. 
углеводного обмена достичь не удавалось. Отмечались частые эпизоды гипергликемии более 30 ммоль/л, эпизоды кетоацидоза, гипогликемическая кома. ДН была выявлена уже на стадии хронической почечной недостаточности через 13 лет от момента дебюта заболевания, через 4 года отмечалось развитие терминальной ХБП. В 2011 г. пациентке была выполнена сочетанная гетеротопическая аллотрансплантация почки и панкреатодуоденального комплекса с кишечным дренированием экзокринного секрета ТПЖ путем формирования дуоденоеюноанастомоза. Была отмечена немедленная функция обоих трансплантатов, эугликемия сразу после реперфузии ТПЖ (отмена инсулинотерапии), нормализация уровня азотистых шлаков к 3-м суткам. Была инициирована стандартная трехкомпонентная иммуносупрессивная терапия, которую пациентка получает по настоящее время.

Состояние ТПЖ: до настоящего времени - удовлетворительная функция трансплантата, гликированный гемоглобин в пределах 4,9-5,3\%, без инсулинотерапии.

Состояние почечного трансплантата: в 2011 г. манифестация пиелонефрита, характеризующегося хроническим рецидивирующим течением (3-4 обострения в год на протяжении 7 лет). С 2018 г. в связи с прогрессированием почечной недостаточности был возобновлен программный гемодиализ. Первично нефрогенная анемия развилась по мере прогрессирования ХБП, после трансплантации показатели гемоглобина нормализовались без терапии, однако впоследствии при прогрессивном ухудшении функции трансплантата антианемическая терапия была возобновлена. Прогрессирование минеральных и костных нарушений при ХБП также отмечалось при снижении функции трансплантата: развитие тяжелого остеопороза с множественными низкотравматичными переломами, агрессивное течение вторичного гиперпаратиреоза, наиболее вероятно, обусловленное дополнительным воздействием иммуносупрессивной терапии наряду с фактом предтрансплантационного применения цинакальцета [4]. Обращает внимание абортивное течение артериальной гипертензии у пациентки - через 6 мес от первичной инициации программного гемодиализа установилась стойкая тенденция к гипотензии, сохраняющаяся, в том числе после возобновления ЗПт, по настоящее время.

Особенностью данного случая явилось развитие возвратной терминальной почечной недостаточности после проведенной СТПиПЖ на фоне сохранной функции ТПЖ, связанной с рецидивирующим течением пиелонефрита трансплантата почки. приведшей к его утрате.

Пациент 3., 44 года, диагноз СД 1 типа был установлен в 1987 г. (с 13-летнего возраста, в течение 31 года). Схематично течение заболевания представлено на рис. 3.

В данном случае также за весь период заболевания компенсация углеводного обмена не достигалась, отмечалась стойкая тенденция к гипергликемии в дебюте заболевания; в анамнезе 4 тяжелые гипогликемии с потерей сознания (в период ЗПт гемодиализом).

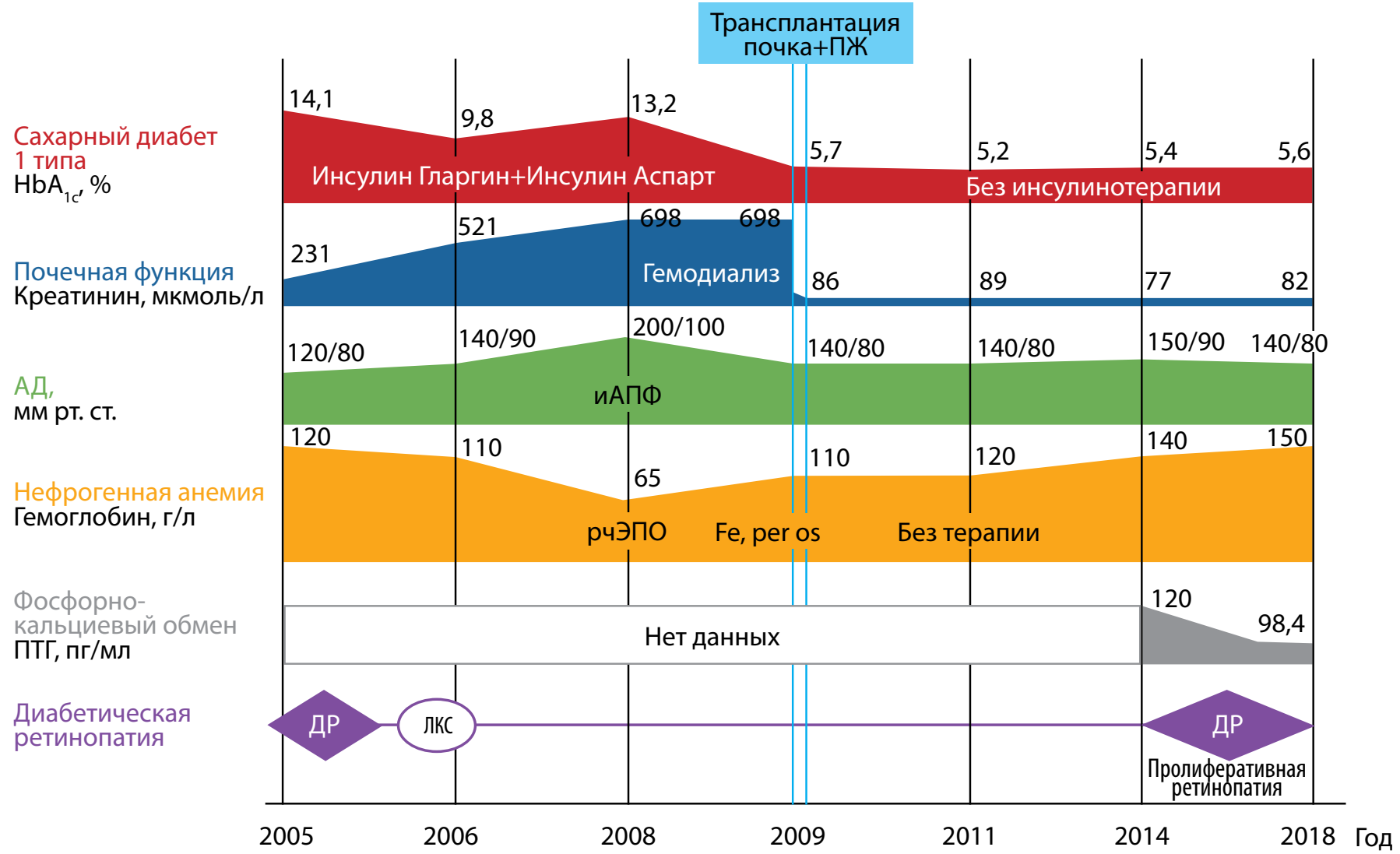

Рис. 3. Графическое представление течения заболевания пациента 3.

$\mathrm{HbA}_{1 c}$ - гликированный гемоглобин; ПТГ - паратгормон; рчЭПО - препараты рекомбинантного человеческого эритропоэтина; Fe per os - пероральные препараты железа; ДР - диабетическая ретинопатия; ЛКС - лазеркоагуляция сетчатки; ФЭК+ИОЛ - факоэмульсификация катаракты+имплантация интраокулярной линзы; иАПФ - ингибиторы ангиотензинпревращающего фермента. 
Регулярный скрининг наличия осложнений СД не проводился. ДН была выявлена в 2005 г. уже на стадии повышения уровня креатинина, наряду с повышением артериального давления (АД) до 150-200/90-100 мм рт. ст., тогда же был инициирован прием иАПФ (несвоевременно). ЗПТ (сначала посредством перитонеального диализа, впоследствии - гемодиализа) - с 2006 г. В 2009 г. была проведена забрюшинная СТПиПЖ с кишечным отведением экзокринного секрета путем формирования дуоденоеюноанастомоза. Была отмечена немедленная функция обоих трансплантатов: эугликемия на 1-е сутки, нормализация уровня азотистых шлаков к 3-м суткам после операции. Была инициирована трехкомпонентная иммуносупрессивная терапия, которую получает по настоящее время.

Состояние ТПЖ: удовлетворительная функция трансплантата по настоящее время. Уровень гликированного гемоглобина стабильно в пределах 5,2-5,7\%

Состояние почечного трансплантата: удовлетворительная функция почечного трансплантата по настоящее время в рамках ХБП С1-2А1 (рСКФ ЕРІ 75-103 мл/мин/1,73 м² соотношение альбумин/креатинин разовой порции мочи 0,82 мг/ммоль).

Нефрогенная анемия была выявлена в 2006 г., получал терапию рекомбинантными человеческими эритропоэтинами (рчЭПО). После проведения аллотрансплантации почки и Пж препарат был отменен в связи с нормализацией уровня гемоглобина, в настоящее время без терапии.

Данные о наличии минеральных и костных нарушений при ХБП отсутствуют до 2007 г., когда впервые было выявлено уменьшение в росте на 0,8 см, снижение костной плотности по DEXA в бедре до -1,2 SD (Z-критерий), по поводу чего получал препараты кальция. В 2014 г. был диагностирован вторичный гиперпаратиреоз, с 2015 г. была инициирована антирезорбтивная терапия, в 2016 г. перелом левого надколенника при падении с высоты собственного роста. Лечение эпизодически приостанавливалось ввиду указания в анализах на гиперкальциемию, далее с начала 2019 г. было возобновлено на постоянной основе после коррекции дефицита витамина D.

После проведения СТПиПЖ по настоящее время отмечается нормализация показателей АД в пределах 110-120/70-80 мм рт. ст.

В 1996 г. была диагностирована пролиферативная диабетическая ретинопатия обоих глаз, проводились неоднократные лазерные коагуляции сетчатки (ЛКС) в 1996, 2002 гг. В настоящее время состояние глазного дна стабильное, оперативного лечения требует катаракта.

Особенностью данного клинического случая является стойкая удовлетворительная функция обоих трансплантатов в результате СТПиПЖ в течение 11 лет. На этом фоне отмечается нормализация показателей АД, уровня гемоглобина, стабилизация состояния глазного дна. Однако обращает на себя внимание формирование язвенного дефекта стопы спустя 9 лет эугликемии, прогрессирование атеросклеротического поражения артерий нижних конечностей, а также катаракты, что может служить подтверждением тому, что достижение эугликемии не является единственным фактором, определяющим благоприятное течение осложнений СД после СТПиПЖ.

\section{ОБСУЖДЕНИЕ}

СТПиПЖ является оптимальным методом лечения пациентов с СД1 с терминальной почечной недостаточностью. Было показано, что ожидаемая продолжительность жизни пациентов после трансплантации на 15 лет больше, чем пациентов, находящихся в листе ожидания, и на 10 лет дольше, чем для пациентов после изолированной трансплантации почки [5]. Более того, СТПиПж значимо улучшает качество жизни и может предотвращать прогрессирование осложнений диабета [6].

Тем не менее, само оперативное вмешательство является крайне сложным и предъявляет высокие требования как к качеству донорского материала, состоянию реципиента, так и к квалификации хирурга. Кроме того, достаточно часто в раннем послеоперационном периоде наблюдаются хирургические осложнения, которые в конечном счете могут привести как к потере одного или обоих трансплантатов, так и к гибели пациента. Наряду с этим даже успешно проведенная операция, немедленная функция трансплантатов, благоприятное течение раннего послеоперационного периода не гарантируют сохранения удовлетворительной функции обоих органов.

Стойкая нормогликемия без необходимости инсулинотерапии после СТПиПЖ является одним из основных факторов улучшения качества жизни для пациентов с СД1. Однако бывают случаи, когда пациенты вновь начинают испытывать потребность в инсулинотерапии в связи с развитием гипергликемии, генез которой может быть различным.

Известно, что после СТПиПЖ антитела к инсулинпродуцирующим клеткам ПЖ могут персистировать, исчезнуть или появиться вновь после некоторого периода их отсутствия [7, 8]. Наличие титра антител перед трансплантацией может выступить предиктором рецидива СД1 впоследствии, предшествуя манифестации гипергликемии на несколько месяцев или даже лет, а также, по данным ряда авторов, иметь некие ассоциации с острым отторжением $[7,9,10]$. Интересной находкой является тот факт, что с более высоким риском повторного развития СД1 ассоциирована именно конверсия антител, а не их текущий статус (положительные или отрицательные), что может служить подтверждением того факта, что, скорее, реактивация гуморального аутоиммунного ответа является до конца не изученным триггером. Таким образом, многим исследователям представляется целесообразным включить тестирование пациентов после СТПиПЖ на антитела в динамическое наблюдение с целью максимально ранней диагностики рецидива СД1 [11, 12]. К сожалению, в случае нашего пациента Н. статус его панкреатических антител неизвестен, и точная причина утраты ТПЖ неизвестна.

С другой стороны, известно, что такролимус, который является основой иммуносупрессивной терапии у большинства пациентов после СТПиПЖ, индуцирует развитие гипергликемии посредством прямой токсичности в отношении бета-клеток и влияния на секрецию инсулина, что впоследствии может имитировать рецидив СД 1 [13].

Получить более полную картину с определением возможной причины гипергликемии в отдаленном периоде после СТПиПЖ может позволить биопсия ТПЖ. 
В настоящее время, ввиду модификации оперативной методики пересадки ПЖ с ее забрюшинным расположением и формированием междуоденального анастомоза, наиболее безопасной с целью диагностики отторжения может явиться биопсия участка слизистой двенадцатиперстной кишки [14]. Проведение биопсии самого ТПж с целью изучения его гистологического строения, включая рецидив СД1 после сочетанной пересадки, описано рядом авторов, однако ее выполнение должно быть регламентировано соотношением риск-польза ввиду высокой опасности утраты трансплантата вследствие хирургических и инфекционных осложнений [15].

Постепенное повышение уровня креатинина, развитие протеинурии и снижение уровня гемоглобина отражают хроническую дисфункцию почечного трансплантата, являющуюся выражением тесного взаимодействия иммунологических и неиммунологических факторов повреждения почечной ткани: исходного состояния пересаженной почки, хронического отторжения, инфекционных агентов, токсичности лекарственных средств, гипертензии, дислипидемии [16].

Потеря трансплантата оказывает существенное влияние на относительный риск (ОР) смерти реципиента, повышая его практически в 11 раз. Инфекции мочевых путей (ИМП) достаточно часто развиваются после трансплантации (35-79\%) и обычно манифестируют в течение первого года после операции, несмотря на профилактику антибиотиками. Известными факторами риска в таком случае являются женский пол, необходимость катетеризации мочевого пузыря, СД, предшествующие ИМП, длительный период диализа до трансплантации, камни в почках и пузырно-мочеточниковый рефлюкс $[17,18]$.

Более того, пиелонефрит трансплантата часто трудно диагностировать, поскольку он нередко может иметь стертое течение. В качестве потенциального механизма влияния обострений ИМП на трансплантат почки считают, что бактериальная инфекция активирует иммунную систему, приводя к острому или хроническому отторжению и последующему снижению почечной функции. Кроме того, вероятно, острый пиелонефрит трансплантата может вызывать развитие интерстициального фиброза, приводя к повреждению почки $[19,20]$. Наконец, тип иммуносупрессии тесно связан с развитием ИМП. Хорошо известно, что иммуносупрессия может влиять на резистентность Enterococcus spp. к $\beta$-лактамным антибиотикам, влияя на экспрессию пенициллинсвязывающих белков (РВР). Во многих исследованиях было показано, что азатиоприн, микофенолата мофетил и антитимоцитарный глобулин ассоциированы с более высокой частотой ИМП в посттрансплантационном периоде, тогда как другие лекарственные средства (ингибиторы кальциневрина, эверолимус), вероятно, не повышают риск, более того, отмена стероидов не оказывает никакого эффекта на риск ИМП [21, 22].

Как видно из представленных клинических случаев, у пациентки Б. имело место развитие дисфункции почечного трансплантата на фоне функционирующего ТПж именно вследствие рецидивирующего пиелонефрита трансплантата. Факторами риска в данном случае служили женский пол, прием иммуносупрессивной терапии, кроме того, нельзя исключить влияние структурных факторов (рефлюкс). То есть в данном случае даже стойкая нормогликемия не смогла существенно уменьшить влияние ИМП на состояние пересаженной почки.

В отношении диабетических осложнений в целом доказано, что функционирующий ТПЖ оказывает протективное воздействие на функцию трансплантата почки. Достижение стойкой нормогликемии не только предотвращает развитие ДН, но также вызывает обратное развитие гистологических повреждений, характерных для ДН [23].

Существуют свидетельства того, что трансплантация Пж и стойкая нормогликемия стабилизируют и даже улучшают состояние глазного дна. Однако у ряда пациентов диабетическая ретинопатия (ДР) может прогрессировать даже в условиях нормогликемии [24], что, вероятно, ассоциировано с выраженной степенью повреждения сетчатки к моменту пересадки.

Относительно нейропатии непосредственно после СТПиПЖ - в некоторых исследованиях отмечается улучшение функций двигательных и чувствительных нервов, что подтверждается улучшением нервной проводимости. Начальные регенеративные изменения тонких немиелинизированных волокон наблюдают при использовании конфокальной микроскопии роговицы в течение первых 6 мес после трансплантации ПЖ [25]. В отношении автономной нейропатии эффект менее очевиден. Ряд авторов отмечают положительный эффект функционирующего ТПЖ в отношении сердечной функции (улучшение фракции выброса, улучшение диастолической функции и др.), а также состояния и выраженности атеросклеротического поражения периферических артерий [26].

Однако обращает на себя внимание некоторое отсроченное развитие благоприятных эффектов СТПиПЖ, что, вероятно, может быть связано с феноменом метаболической памяти [27]. Ключевым патогенетическим звеном является запуск процесса окислительного стресса под длительным воздействием гипергликемии с формированием пула конечных продуктов гликирования. Это приводит к образованию соединений, модифицирующих белки и длительно сохраняющихся в организме, несмотря на устранение первопричинного фактора (гипергликемия). В ряде случаев достижение стойкой эугликемии в результате СТПиПЖ позволяет снизить интенсивность уже запущенного развития сосудистых осложнений и осложнений ХБП. Напротив, у ряда реципиентов деструктивные процессы продолжают свое развитие, значимо усугубляясь воздействием иммуносупрессивной терапии $[28,29]$. Так, у нашего пациента 3. даже через 9 лет после успешной СТПиПЖ отмечались формирование язвенных дефектов стоп, отрицательная динамика атеросклеротического поражения артерий нижних конечностей, прогрессирование катаракты (в том числе, вероятно, в результате влияния иммуносупрессивной терапии), а также минеральных и костных нарушений при ХБП (снижение плотности костной ткани на фоне вторичного гиперпаратиреоза).

\section{ЗАКЛЮЧЕНИЕ}

Таким образом, СТПиПЖ является радикальным хирургическим методом лечения пациентов с СД1 и тер- 
минальной стадией почечной недостаточности, позволяющим достичь стойкой эугликемии и нормализации почечной функции. Однако нормогликемия как таковая не является гарантией стабилизации состояния пациентов. Необходимо тщательно контролировать многочисленные классические факторы (АД, липидный спектр, анемию, длительность диализного периода, качество донорского материала, выраженность других осложнений СД, состояние сердечно-сосудистой системы, стабильность концентрации иммуносупрессивных препаратов, осложнения иммуносупрессивной терапии, сопутствующие инфекции) с целью максимального сохранения функции обоих трансплантатов. Накопление клинического опыта доказывает необходимость изучения протеомных, иммунологических, эпигенетических аспектов патогенеза диабетических осложнений и осложнений ХБП, поскольку изолированная компенсация гипергликемии и уремии, несмотря на их превалирующее значение, не в состоянии обеспечить стойкую стабилизацию в долгосрочной перспективе.

\section{ДОПОЛНИТЕЛЬНАЯ ИНФОРМАЦИЯ}

Финансирование работы. Работа проведена в рамках выполнения Государственного задания Минздрава России (АААA-A20-120011790181-1).
Конфликт интересов. Авторы декларируют отсутствие явных и потенциальных конфликтов интересов, связанных с публикацией настоящей статьи.

Участие авторов. Северина А.С. - лечение пациентов, концепция и дизайн статьи, анализ литературы, редактирование текста, написание статьи, утверждение итогового варианта текста рукописи; Ларина И.И. лечение пациентов, анализ литературы, написание статьи, утверждение итогового варианта текста рукописи; Шутова А.С. - лечение пациентов, анализ литературы, формирование первичного варианта текста рукописи, графическое оформление случаев; Шамхалова М.Ш. - редактирование текста, утверждение итогового варианта текста рукописи; Дмитриев И.В. - хирургическое лечение пациентов, редактирование текста, утверждение итогового варианта текста рукописи; Пинчук А.В. хирургическое лечение пациентов, утверждение итогового варианта текста рукописи; Арзуманов С.В. - хирургическое лечение пациентов, утверждение итогового варианта текста рукописи; Шестакова М.В. редактирование текста; утверждение итогового варианта текста рукописи. Все авторы внесли существенный вклад в проведение исследования и подготовку статьи, прочли и одобрили финальную версию перед публикацией.

Благодарности. Выражается благодарность всем пациентам, участвовавшим в данном исследовании, а также сотрудникам ФГБУ «НМИЦ эндокринологии», ГБУз «НИИ СП им. Н.В. Склифосовского ДЗМ», НИИ урологии и интервенционной радиологии им. Н.А. Лопаткина филиал ФГБУ «НМИЦ радиологии», участвовавшим в лечении и обследовании пациентов.

\section{СПИСОК ЛИТЕРАТУРЫ | REFERENCES}

1. Gruessner AC, Sutherland DE. Pancreas-transplant outcomes for United States (US) cases as reported to the United Network for Organ Sharing (UNOS) and the International Pancreas Transplant Registry (IPTR). New York: Oxford University Press. 2010;299-315. doi: https://doi.org/10.1093/med/9780199565863.003.0017

2. Lodhi SA, Lamb KE, Meier-Kriesche HU. Solid organ allograft survival improvement in the United States: the long-term does not mirror the dramatic short-term success. Am J Transplant. 2011;11(6):1226-1235. doi: https://doi.org/10.1111/j.1600-6143.2011.03539.x

3. Seron D, Moreso F, Arias M, et al. Estimation of renal allograft halflife: fact or fiction? Nephrol Dial Transplant. 2011;26(9):3013-3018. doi: https://doi.org/10.1093/ndt/gfa788

4. Wolf $\mathrm{M}$, Weir MR, Kopyt $\mathrm{N}$, et al. Prospective cohort study of mineral metabolism after kidney transplantation. Transplantation. 2016;100(1):184-189. doi: https://doi.org/10.1097/TP.0000000000000823

5. Viglietti D, Serrato T, Abboud I, et al. Kidney graft dysfunction in simultaneous pancreas-kidney recipients after pancreas failure: analysis of early and late protocol biopsies. Clin Transplant. 2013;27(3):E249-255. doi: https://doi.org/10.1111/ctr.12095

6. Pera IP, Vasallo MJ, Rabasa TA, et al. Quality of life in simultaneous pancreas-kidney transplant recipients. Clin Transplant. 2009;23(5):600-605. doi: https://doi.org/10.1111/j.1399-0012.2009.01054.x

7. Vendrame F, Hopfner YY, Diamantopoulos S, et al. Risk factors for type 1 diabetes recurrence in immunosuppressed recipients of simultaneous pancreas-kidney transplants. Am J Transplant. 2016;16(1):235-245. doi: https://doi.org/10.1111 / ajt.13426

8. Martins L, Malheiro J, Henriques AC, et al. Pancreaskidney transplantation and the evolution of pancreatic autoantibodies. Transplant Proc. 2009;41(3):913-915. doi: https://doi.org/10.1016/j.transproceed.2009.01.068

9. Martins LS. Autoimmune diabetes recurrence should be routinely monitored after pancreas transplantation. World J Transplant 2014;4(3):183-187. doi: https://doi.org/10.5500/wjt.v4.i3.183

10. Vendrame F, Pileggi A, Laughlin E, et al. Recurrence of type 1 diabetes after simultaneous pancreas-kidney transplantation, despite immunosuppression, is associated with autoantibodies and pathogenic autoreactive CD4 T-cells. Diabetes. 2010;59(4):947-957. doi: https://doi.org/10.2337/db09-0498
11. Krischer JP, Cuthbertson DD, Yu L, et al. Screening strategies for the identification of multiple antibody-positive relatives of individuals with Type 1 diabetes. J Clin Endocrinol Metab. 2003;88(1):103-108. doi: https://doi.org/10.1210/jc.2002-020760

12. Wenzlau JM, Juhl K, Yu L, et al. The cation efflux transporter ZnT8 (SIc30A8) is a major autoantigen in human type 1 diabetes. Proc Natl Acad Sci U S A. 2007;104(43):17040-17045. doi: https://doi.org/10.1073/pnas.0705894104

13. Trinanes J, Rodriguez-Rodriguez AE, Brito-Casillas Y, et al. Deciphering ta- crolimus-induced toxicity in pancreatic p cells. Am J Transplant. 2017;17(11):2829-2840. doi: https://doi.org/10.1111/ajt.14323

14. Сторожев Р.В., Нефедова Г.А., Тетерин Ю.С., и др. Гистологическое изучение биоптатов слизистой оболочки донорской двенадцатиперстной кишки в диагностике отторжения панкреатодуоденального комплекса: опыт НИИ СП им. Н.В. Склифосовского // Трансплантология. 2018. - T. 10. - №2. - C. 110-117. [Storozhev RV, Nefedova GA, Teterin YuS, et al. Histological study of donor's duodenal mucosa biopsy specimens in the diagnosis of pancreatoduodenal complex rejection: the experience of N.V. Sklifosovsky Research Institute for Emergency Medicine. Transplantologiia. 2018;10(2):110-117. (In Russ.)]. doi: https://doi.org/10.23873/2074-0506-2018-10-2-110-117

15. Martin-Pagola A, Sisino G, Allende G, et al. Insulin protein and proliferation in ductal cells in the transplanted pancreas of patients with Type 1 diabetes and recurrence of autoimmunity. Diabetologia. 2008;51(10):18031813. doi: https://doi.org/10.1007/s00125-008-1105-x

16. Gourishankar S, Halloran PF. Late deterioration of organ transplants: a problem in injury and homeostasis. Curr Opin Immunol. 2002;14(5):576-583. doi: https://doi.org/10.1016/s0952-7915(02)00386-2

17. Adamska Z, Karczewski M, Cichanska L, et al. Bacterial infections in renal transplant recipients. Transplant Proc. 2015;47(6):1808-1812. doi: https://doi.org/10.1016/j.transproceed.2015.03.046

18. Lim JH, Cho JH, Lee JH, et al. Risk factors for recurrent urinary tract infection in kidney transplant recipients. Transplant Proc. 2013;45(4):1584-1589. doi: https://doi.org/10.1016/j.transproceed.2012.12.011 
19. Bodro M, Sanclemente G, Lipperheide I, et al. Impact of urinary tract infections on short-term kidney graft outcome. Clin Microbiol Infect. 2015;21(12):1 104.e1-8. doi: https://doi.org/10.1016/j.cmi.2015.07.019

20. Dupont PJ, Psimenou E, Lord $\mathrm{R}$, et al Late recurrent urinary tract infections may produce renal allograft scarring even in the absence of symptoms or vesicoureteric reflux. Transplantation. 2007:84(3):351-355. doi: https://doi.org/10.1097/01.tp.0000275377.09660.fa

21. Chuang P, Parikh CR, Langone A. Urinary tract infections after renal transplantation: a retrospective review at two US transplant centers. Clin Transplant. 2005;19(2):230-235. doi: https://doi.org/10.1111/j.1399-0012.2005.00327.x

22. Alangaden GJ, Thyagarajan R, Gruber SA, et al. Infectious complications after kidney transplantation: current epidemiology and associated risk factors. Clin Transplant. 2006;20(4):401-409. doi: https://doi.org/10.1111/j.1399-0012.2006.00519.x

23. Fioretto P, Steffes MW, Sutherland DE, et al. Reversal of lesions of diabetic nephropathy after pancreas transplantation. N Eng/ J Med. 1998;339(2):69-75. doi: https://doi.org/10.1056/NEJM199807093390202

24. Kim Y, Shin S, Han D, et al. Long-term effects of pancreas transplantation on diabetic retinopathy and incidence and predictive risk factors for early worsening. Transplantation. 2018:102(1):e30-e38. doi: https://doi.org/10.1097/TP.0000000000001958

25. Tavakoli M, Mitu-Pretorian M, Petropoulos IN, et al. Corneal confocal microscopy detects early nerve regeneration in diabetic neuropathy after simultaneous pancreas and kidney transplantation. Diabetes. 2013;62(1):254-260 doi: https://doi.org/10.2337/db12-0574

26. Lindah JP, Hartmann A, Aakhus S, et al. Long-term cardiovascular outcomes in type 1 diabetic patients after simultaneous pancreas and kidney transplantation compared with living donor kidney transplantation. Diabetologia. 2016;59(4):844-852. doi: https://doi.org/10.1007/s00125-015-3853-8

27. Ceriello A, Ihnat M, Thorpe J. Clinical review 2: The «metabolic memorys: is more than just tight glucose control necessary to prevent diabetic complications? J Clin Endocrinol Metab. 2016;94(2):410-415. doi: https://doi.org/10.1210/jc.2008-1824

28. Yamagishi S, Nakamura N, Suematsu M, et al. Advanced glycation end products: a molecular target for vascular complications in diabetes. Mol Med. 2015;21(Suppl 1):S32-40. doi: https://doi.org/10.2119/molmed.2015.00067

29. Koyama H, Nishizawa Y. AGEs/RAGE in CKD: irreversible metabolic memory road toward CVD? Eur J Clin Invest. 2010;40(7):623-635 doi: https://doi.org/10.1111/j.1365-2362.2010.02298

\section{ИНФОРМАЦИЯ ОБ АВТОРАХ [AUTHORS INFO]}

*Ларина Ирина Игоревна, н.c. [Irina I. Larina, MD, research associate]; адрес: Россия, 117036 Москва, ул. Дм. Ульянова, д. 11 [address: 11 Dm. Ulyanova street, 117036 Moscow, Russian Federation]; ORCID: http://orcid.org/0000-0001-6783-4200; eLibrary SPIN: 1220-6080; e-mail: irina.larina1993@mail.ru

Северина Анастасия Сергеевна, К.м.н., В.н.с. [Anastasia S. Severina MD, PhD, leading research associate]; ORCID: https://orcid.org/0000-0002-0296-4933; eLibrary SPIN: 3182-9510; e-mail: ansev1@mail.ru

Шутова Александра Сергеевна, клинический ординатор [Aleksandra S. Shutova, MD, clinical resident]; ORCID: https://orcid.org/0000-0003-0047-7223; eLibrary SPIN: 4774-0114; e-mail: shutova.aleksandra@gmail.com

Шамхалова Минара Шамхаловна, д.м.н. [Minara S. Shamhalova, MD, PhD];

ORCID: https://orcid.org/0000-0002-3433-0142; eLibrary SPIN: 4942-5481; e-mail: shamkhalova@mail.ru

Дмитриев Илья Викторович, к.м.H. [llya V. Dmitriev, MD, PhD]; ORCID: https://orcid.org/0000-0002-5731-3310; eLibrary SPIN: 3916-6591; e-mail: ildmi@mail.ru

Пинчук Алексей Валерьевич, к.M.н. [Aleksey V. Pinchuk, MD, PhD]; ORCID: https://orcid.org/0000-0001-9019-9567; eLibrary SPIN: 8875-2456; e-mail: avpin@rambler.ru

Арзуманов Сергей Викторович, д.м.н. [Sergey V Arzumanov, MD, PhD]; ORCID: https://orcid.org/0000-0001-7358-3364; eLibrary SPIN: 8317-3723; e-mail: kidneytranspl@gmail.com

Шестакова Марина Владимировна, д.М.Н., профессор, академик PAH [Marina V. Shestakova MD, PhD, Professor]; ORCID: https://orcid.org/0000-0002-5057-127X; eLibrary SPIN: 7584-7015; e-mail: nephro@endocrincentr.ru

\section{ЦИТИРОВАТЬ:}

Северина А.С., Ларина И.И., Шутова А.С., Шамхалова М.Ш., Дмитриев И.В., Пинчук А.В., Арзуманов С.В., Шестакова М.В. Сочетанная трансплантация почки и поджелудочной железы при сахарном диабете 1 типа. Серия наблюдений // Сахарный диабет. - 2020. - T. 23. — №3. - C. 275-282. doi: https://doi.org/10.14341/DM12509

\section{TO CITE THIS ARTICLE:}

Severina AS, Larina II, Shutova AS, Shamkhalova MS, Dmitriev IV, Pinchuk AV, Arzumanov SV, Shestakova MV. Simultaneous pancreas-kidney transplantation in type 1 Diabetes Mellitus. Clinical options. Diabetes Mellitus. 2020;23(3):275-282. doi: https://doi.org/10.14341/DM12509 